\title{
Prototipagem de modelo de redes para identificar relações principais no desenvolvimento da vacina para a COVID-19
}

\author{
Guilherme Pedó Flores; \\ Wyllians Vendramini Borelli; \\ Filipe Campelo Xavier da Costa
}

\section{resumo:}

A pandemia subsequente à doença pelo novo-Coronavirus-2019 (COVID-19) exige medidas imediatas, como uma vacina, para evitar o aumento de mortes em decorrência da doença. A contribuição do design estratégico, através da abordagem de redes, pode auxiliar no planejamento e na facilitação do desenvolvimento da vacina. O objetivo deste trabalho é identificar os atores principais no desenvolvimento da vacina para COVID-19 e propor um modelo de redes para analisar as relações entre os diferentes atores. Os atores-chave identificados foram, nomeadamente, a academia, o governo, as entidades internacionais e a sociedade. O modelo foi desenvolvido com auxílio da ferramenta Miro, representando diversos atores principais e atores secundários como nós grandes e pequenos, respectivamente. As relações entre os nós foram representadas através de conexões, sendo a distância e a força entre as conexões também descritas. Em suma, foram identificadas relações entre academia-governo e governo-sociedade como fundamentais para o desenvolvimento da vacina para COVID-19.

\section{palavras-chave:}

Design estratégico; Redes; Vacina; Codesign; Doenças 


\section{Introdução}

O surto causado pela doença do novo-Coronavírus-2019 (COVID-19) foi súbito e inesperado na maioria dos países. Os primeiros casos foram no fim de Dezembro de 2019, subsequentemente declarada a pandemia pela OMS em Março de 2020 (CUCINOTTA; VANELLI, 2020). A evolução da COVID-19 continua imprevisível, e esta característica é exacerbada pela heterogeneidade dos sistemas de saúde no mundo e pelas dificuldades em obter dados precisos da infecção e de sua imunização. Em virtude da magnitude desta pandemia, a maioria dos países adotou o isolamento social como estratégia de contenção desta doença.

A quarentena foi descrita como o meio mais seguro e eficaz para diminuir o contágio da COVID-19 (CONTI et al., 2020). Dentre vários medicamentos usados para tratamento e fórmulas integrativas para prevenção desta doença, nenhuma se comprovou eficaz como tratamento-alvo contra o vírus causador da COVID-19. Assim, o cenário devastador da doença toma curso, atingindo uma proporção maior de pessoas e gerando um número crescente de mortes ao redor do mundo. Até o dia 23 de julho, a COVID-19 causou a morte de mais de 620.000 pessoas no mundo, sendo 85.000 apenas em território brasileiro (CONTROL, 2020b; SAÚDE, 2020). Diante deste cenário, pesquisadores integrados a governos ao redor do mundo iniciaram a busca para a prevenção eficaz e efetiva contra a COVID-19 através do desenvolvimento da imunoprofilaxia contra a doença, também chamada de vacinação. O propósito dessa vacina é estimular o corpo a desenvolver imunidade e evitar o quadro patológico da COVID-19, por meio de uma manipulação do vírus, ou seus componentes imunogênicos, e exposição do indivíduo a suas partes. Neste contexto, a vacina é proposta como uma solução lato sensu para a epidemia atual. No entanto, o desenvolvimento da vacina é complexo, exigindo a integração de diversas dimensões sociais, em uma abordagem colaborativa e coordenada com empresas, governo, academia, sociedade, entre outros (HAMED, 2020).

A prática de design trabalha com "problemas caóticos" (SCHÖN, 2000), ou seja, problemas práticos, reais, cujas soluções não se dão por meio de teorias e técnicas já conhecidas. Para aprender a solucionar problemas dessa natureza, faz-se necessária uma abordagem baseada nas perspectivas do design estratégico na busca de soluções para problemas complexos, como o desenvolvimento da imunoprofilaxia para COVID-19. O design estratégico per se é a aplicação dos princípios do design orientados em perspectivas futuras de um cenário (HOLSTON, 2011). Usualmente dirigido para empresas e contexto industrial, o design estratégico possui um potencial enorme de aplicação para sistemas complexos - como a saúde. O desenvolvimento de novas moléculas, tanto para prevenção quanto para tratamento, é uma necessidade diária que interage de maneira multifacetada com uma miríade de setores da sociedade. Justamente por ter a resolução de problemas em seu âmago, a implementação do design estratégico para o contexto de saúde tem grande potencial inovador para facilitar e agilizar o avanço dos processos nesta área.

A abordagem em redes é uma ferramenta proposta pelo design estratégico para solução de problemas complexos como o desta pandemia por COVID-19. A sociedade, em geral, é formada por redes complexas de interação social, nas quais os indivíduos se agrupam de maneira natural para colaboração e auxílio conjunto para resolução de problemas (CASTELLS, 2002). Estas redes são compostas por nós, ligadas através de conexões, as tem características dinâmicas e mutáveis. No desenvolvimento de imunoprofilaxia para COVID-19, uma série de redes podem ser identificadas, tanto a nível biológico, quanto a nível populacional-governamental. Além disso, um dos pilares do design estratégico, o codesign, visa engajar sistematicamente diversos atores na solução de um problema de interesse comum (MERONI, 2008). Propõe-se um olhar para esse assunto que pode contribuir para um pensamento mais colaborativo no processo de criação de soluções para este novo cenário causado pelo COVID-19. Neste contexto, o objetivo deste trabalho é identificar os principais atores do processo de desenvolvimento da imunoprofilaxia COVID-19 e propor, sob a ótica do design estratégico, uma abordagem de redes complexas para analisar as relações entre os atores. 


\section{Identificação dos atores-chave no desenvolvimento da vacina para COVID-19}

A identificação de atores busca promover em caráter colaborativo o diálogo estratégico para catalisar e orientar a pluralidade de atores envolvidos na solução do problema, através da interpretação da visão compartilhada. O processo de desenvolvimento de uma vacina na atual pandemia COVID-19 precisa ser fundamentado em três pilares: velocidade, produção e distribuição em larga escala, e acesso global (YAMEY et al., 2020). De acordo com o Centro para Controle de Doenças (em inglês, CDC), várias etapas são necessárias para desenvolver uma vacina (CONTROL, 2020a). O processo envolve uma gama de atores articulados desde o conceito da vacina até sua disponibilização para o mercado. Simplificadamente, o processo se inicia com o estágio exploratório de identificação do componente de imunoprofilaxia, avançando para testes pré-clínicos, desenvolvimento clínico, revisão regulatória e aprovação, produção em série e finalmente o controle de qualidade deste produto. Usualmente, este processo é lento e barra em uma série de dificuldades, levando em torno de 10-12 anos para sua finalização (PRONKER et al., 2013) - o que a torna teoricamente inviável em um período de pandemia.

A academia, representada por pesquisadores e cientistas, é reconhecida como o fator inicial neste processo por entender a necessidade da sociedade e propor alternativas para solucioná-lo - a nível biológico. Este nível do desenvolvimento da vacina é dividido em 3 partes: a identificação dos candidatos à vacina, os ensaios pré-clínicos e os ensaios clínicos (em 3 diferentes fases). No primeiro estágio da academia, ocorre a identificação de candidatos a vacina - desde fragmentos de análise genética até fragmentos do vírus inativo ou atenuado (THANH LE et al., 2020). As linhas de pesquisa conduzidas por cientistas são formadas por grupos de pesquisadores de diferentes níveis que atuam diferentemente: o sênior, com o planejamento e aquisição de financiamento; o júnior, com a técnica do trabalho. Em seguida, os ensaios pré-clínicos, são conduzidos estudos em animais para esclarecimento do mecanismo protetor e da interação doença-hospedeiro (CUNNINGHAM et al., 2016). Estes estudos demonstram o potencial efeito tóxico e a dosagem estimada para uso clínico, nas etapas subsequentes. As fases clínicas são conduzidas por equipes de médicos, enfermeiros e profissionais com treinamento para contato direto com paciente. Os estudos se propõem a testar segurança e reação em humanos (fase I), o impacto de múltiplas variáveis no corpo humano (fase II), e o efeito da formulação final (fase III), para então seguir na aprovação por órgãos regulatórios.

O governo e as agências governamentais de saúde têm uma posição fundamental no desenvolvimento de vacinas. Tais órgãos fornecem financiamento para pesquisas nas fases descritas previamente, e também agem na legislação para permitir ou proibir o uso de novos fármacos ou drogas. Além disso, entidades públicas podem agir com métodos inovadores e impactar a população de maneira rápida e muito eficiente, como a recente criação de centro para produção de vacinas no Reino Unido (PARTRIDGE, 2020). O planejamento de sistemas nacionais para a distribuição das vacinas é uma etapa subsequente à produção em massa.

Entidades internacionais, como a Organização Mundial da Saúde (OMS), tem papel planejador para articular a dinâmica global no desenvolvimento de vacinas. O papel dessas agências é evitar a concentração de vacinas em países com mais renda, o que gera desigualdade nos sistemas de saúde do mundo (FIDLER, 2011). É também dever dessas entidades zelar por um sistema de alocação justo na distribuição das vacinas. Atualmente, as entidades internacionais pouco auxiliam na distribuição de financiamentos ou distribuição de conhecimento neste contexto (YAMEY et al., 2020). Além disso, a abrangência desses órgãos permite sua atuação na logística da distribuição de avanços em saúde de maneira menos desigual.

Por último, o apoio da sociedade se faz necessário para o uso da vacina. A desconfiança em relação a vacinas é um movimento recente, mesmo que niilista. A confiança da população no uso da vacina é um fator fundamental para o desenvolvimento da mesma. No entanto, a maioria das pessoas relata desejo em receber a vacina assim que esta estiver disponível (SCHAFFER DEROO; PUDALOV; FU, 2020). Estudos referem que a "imunidade de rebanho", ou seja, quando a sociedade adquire um nível de proteção que diminui significativamente sua disseminação, ocorre quando $70 \%$ dos indivíduos estão imunes a doença - vacinados, neste caso (KWOK et al., 2020). 


\section{A construção de cenários futuros operados por redes para a solução de problemas complexos}

O gráfico de redes prototipado busca acolher, através da fenomenologia, a riqueza de expressões e, para os nossos fins, alguns aspectos recorrentes (ZURLO, 2010). Entre esses: sua dimensão situada (dependente da operacionalidade dos objetivos e das circunstâncias da ação); a capacidade de habilitar, com as próprias capacidades, um processo de diálogo entre vários atores; a exigência em satisfazer necessidades diferentes obtendo resultados (reconhecidos) de valor. Dessa forma, o fechamento e a autonomia são essenciais, partindo da abertura necessária para remontar as peças de um âmbito disciplinar fragmentado e, justamente, complexo.

Meroni (2008) assevera que o design estratégico estabelece uma orientação através de cenários para definir suas ações. Os cenários captam os sinais do ambiente e os organizam de forma coerente, imaginando futuros possíveis ou desejáveis. Eles constituem narrativas desses mundos futuros e têm a capacidade de se adaptar às mudanças do ambiente. Dessa forma, os conceitos acima apresentados, são úteis para entendermos como a palavra cenário está sendo empregada neste artigo, como por exemplo na habilidade humana de construir mundos possíveis, antecipar o tempo e postular futuros. $\mathrm{O}$ processo do design deve ser colaborativo, com envolvimento sistemático das partes interessadas (MERONI, 2008)

Dados os atores descritos previamente, a abordagem em rede contribui de maneira substancial em propor a solução destes problemas (MIELE et al., 2019). Para tanto, é proposto um modelo de figura em duas dimensões, construído através da ferramenta on-line Miro (https://miro.com/). Neste modelo, a representação entre os atores foi feita através de grandes nós, sendo também representados os atores secundários como pequenos nós internos (Figura 1). As conexões são os linhas entre os nós, representando também a força das relações entre os nós. Além disso, a distância entre os nós foi representada proporcionalmente à proximidade das relações entre os atores, sejam eles chaves ou secundários.

$\mathrm{Na}$ prototipagem da figura, os quatro grandes nós foram compostos pelos atores-chaves (academia, governo, entidades internacionais e sociedade) e suas conexões. Cada ator-chave é representado através de uma cor: Academia em amarelo; Governo em lilás; Entidades Internacionais em azul; e Sociedade em verde. Os atores secundários, identificados como importantes para o entendimento da complexidade da rede, foram identificados como pequenos nós internamente aos seus grandes nós. Importantemente, o tamanho de cada grande nó e pequeno nó foram determinados de acordo com o número de conexões e sua importância no cenário de desenvolvimento da vacina para COVID-19. Assim, a Academia foi composta de 3 pequenos nós; o Governo foi composto por 5 pequenos nós; as Entidades Internacionais foram compostas por 3 pequenos nós; e a Sociedade foi composta por 3 pequenos nós.

As conexões entre os nós foram representadas através das linhas com espessuras diferentes. A causalidade entre os nós foi representada por setas direcionais, as quais podem ser bi ou unidirecionais. O tamanho das linhas evidencia a distância entre os grandes nós e os pequenos nós. As conexões entre os pequenos nós de atores-chave diferentes são representadas por linhas tracejadas inter-nós, quando foram fortes o suficiente. Outra característica das conexões é a força da conexão, evidenciada pela espessura da linha. Em suma, totalizaram duas conexões na Academia (sendo 2 conexões unidirecionais dentro do nó de pesquisa clínica); 5 conexões no Governo; 3 conexões nas Entidades Internacionais; e 3 conexões na Sociedade. 


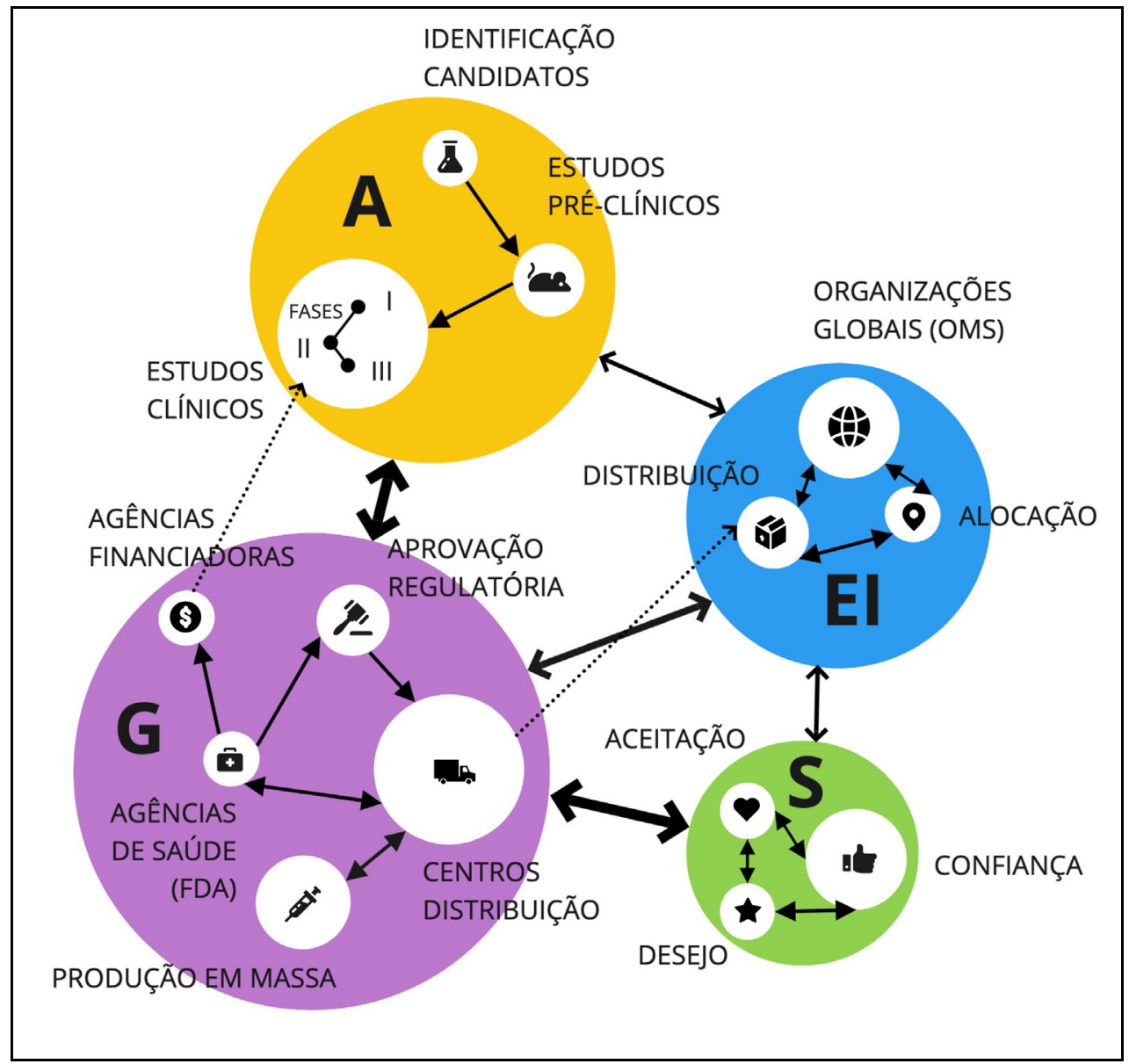

FIGURA 1. Rede de atores identificados no desenvolvimento de uma vacina para COVID-19. Os nós maiores representam os atores-chave, enquanto os nós menores representam os atores secundários. As relações são representadas pelas conexões entre os nós. A: Academia; G: Governo; EI: Entidades Internacionais; S: Sociedade.

\section{Análise dos componentes da rede e suas conexões}

As organizações envolvem múltiplos atores internos e externos para a resolução de tarefas complexas (MANZINI; JÉGOU, 2006). A prototipagem do modelo de rede representado na figura 1 permite a identificação dos atores-chave, atores secundários e das relações complexas existente entre eles. Os atores-chave descritos foram a academia, o governo, as entidades internacionais e a sociedade. Dentre as relações identificadas através da figura 1, as principais foram relacionadas às conexões entre os grandes nós, especificamente academia-governo, academia-entidades internacionais, academiasociedade, governo-sociedade, governo-entidades internacionais e entidades internacionais-sociedade.

O uso do cenário de redes para identificação de atores é uma atividade do design relacionada ao sistema-produto (MERONI, 2008). A construção de cenários de redes possui uma metodologia complexa, a qual foi simplificada devido à urgência de soluções para um contexto de emergência, como a pandemia por COVID-19 (GIUNTA et al., 2017). No entanto, a interpretação das redes permite identificar de maneira visual e precisa os atores e suas relações, pouco interferindo no resultado final. Por meio das redes, é possível também identificar os Papeis atribuídos aos atores, como uma generalização das suas funções no contexto integrado. Este modelo parece, portanto, contribuir fortemente em chamar atenção para a natureza relacional das práticas sociais e organizacionais - e potencialmente o poder entre elas - entre os atores. Um exemplo típico é o papel que a administração pública tem na distribuição de tarefas em uma rede de responsabilidades em inovação, com foco nos interesses público da sociedade (GIUNTA et al., 2017). 
A abordagem do cenário de redes proposta neste trabalho ainda é pouco vista em sistemas de saúde. O design estratégico muito tem a contribuir para o campo da medicina em relação a novas abordagens de problemas complexos, como as interações neuronais (BASSETT; SPORNS, 2017). A ação projetual do design estratégico está intrinsecamente ligada à inovação e à transdisciplinaridade para a elaboração de propostas que promovam a transformação social (ZURLO, 2010). O sucesso dessa abordagem, portanto, gera valor para a sociedade. Além disso, as estratégias de Exploring Design tem sido mencionadas como especialmente úteis na ausência do foco mercadológico, especialmente implementando a geração de processos e sistema-produto (LERMA; DAL PALÙ; DE GIORGI, 2015).

A interação entre academia e governo foi descrita como estreita e forte. Os motivos decorrentes desta classificação são devidos ao fato do íntimo contato da inovação produzida pela academia e da necessidade da aprovação entre os órgãos regulatórios para, de fato, que a inovação possa ser distribuída para a população. Desta forma, os agentes da academia (pesquisadores, cientistas) criam a vacina, e o governo é responsável por criar a legislação necessária para a aprovação, regulação e ampla distribuição dela. Além disso, o governo é um grande patrocinador da academia através do financiamento por meio de fundos de pesquisa e editais para linhas de pesquisa, as quais devem desenvolver e testar a criação de novos tratamentos e estratégias de prevenção. A vacina produzida pela Universidade de Oxford, por exemplo, teve amplo apoio do governo, que indubitavelmente teve apoio fundamental no processo de desenvolvimento (academia) e no processo de distribuição (governo) (SHARPE et al., 2020).

O governo está em íntimo contato com a sociedade. Tanto pela proximidade, quanto pela força da conexão, essa relação se mostra consistente e mutuamente sinérgica. O governo é do povo, para o povo. Cabe ao governo a distribuição da vacina em grandes centros de distribuição, conforme descrito previamente. À sociedade, consequentemente, cabe a necessidade da vacina - inegável dadas as taxas crescentes de mortalidade devido ao COVID-19. Além disso, cabe à sociedade a confiança e o desejo de aceitar a aplicação da vacina logo após seu desenvolvimento. É importante salientar que esses fatores são decisivos no desenvolvimento da imunidade comunitária, protetiva até mesmo para aqueles que não utilizaram a vacina (KWOK et al., 2020).

Governo e entidades internacionais foram representadas como grandes nós de alta relação mútua. No entanto, a maior limitação nesta relação se dá através da limitação entre os atores necessitando uma grande interface de contato para decisões importantes para a população. É evidente que as entidades internacionais tem grandes responsabilidades, mas pouco podem realizar perante a monopolização de recursos (YAMEY et al., 2020).

A academia e entidades internacionais foram representadas de maneira fraca e distante. Esta representação ocorreu devido a pouca perspectiva de avanço por meio dessa interação. As colaborações internacionais presentes na academia impactam diretamente o desenvolvimento da vacina. No entanto, estas colaborações pouco impactam na sua distribuição ou na alocação de recursos, visto que o conhecimento é amplamente divulgado pela comunidade científica internacional de maneira uniforme. Da mesma forma, academia e sociedade são mutuamente relacionadas de maneira importantíssima, mas pouco relevantes para o desenvolvimento da vacina para COVID-19. Cientistas e pesquisadores da academia têm papel fundamental na divulgação de conhecimento científico de maneira acessível à sociedade. A sociedade, consequentemente, beneficia-se dos avanços da academia, como a vacina. As entidades internacionais e a sociedade pouco interagem neste cenário de desenvolvimento de desenvolvimento da vacina.

\section{Conclusões}

Em suma, a complexidade do desenvolvimento da vacina para COVID-19 demonstra uma série de desafios. Os diferentes atores-chave para o processo foram identificados, sendo eles resumidamente descritos como (1) academia, (2) governo, (3) entidades internacionais e (4) a sociedade. Indubitavelmente, uma diversidade de outros atores é identificada dentro dos atores-chave, com interações dinâmicas e mutáveis. A interação desses fatores foi exemplificada através de modelo de 
redes proporcionado pela abordagem do design estratégico, onde se tem o detalhamento de cada ator e das relações existentes entre eles. Neste modelo, os componentes gráficos descrevem as relações através do tamanho de cada nó, a distância entre os atores, a força das conexões e o que elas representam entre os nós. As conexões que mais se destacaram na análise da figura foram academiagoverno e governo-sociedade, devido às suas altas interações. Além disso, ambas relações são essenciais e determinantes no processo de desenvolvimento de uma vacina para COVID-19.

Prototyping of a network modelling to identify major relations in the development of a COVID-19 vaccine

\section{Abstract:}

The pandemic subsequent to the novel-Coronavirus Disease 2019 (COVID-19) demands immediate measures, such as a vaccine, to avoid the increasing number of deaths caused by the disease. The contribution provided by strategic design, through the network approach, may help to plan and facilitate the development of a vaccine for COVID-19. The aim of this study was to identify the major actors in the development of a COVID-19 vaccine, and to propose a network model to analyse the relation between these different actors. Identified key-actors were as follows: the academia, the government, international entities and the society. This model was developed using the tool Miro, in which many principal and secondary actors were represented with big and small hubs, respectively. The relations between these hubs were also represented with edges, in which the distance and the strength of each edge were also described. In sum, the relation between academia-governemnt and government-society were the most important for the development of a COVID-19 vaccine.

Keywords: Strategic design; Network; Vaccine; Codesign; Diseases

\section{Referências bibliográficas}

BASSETT, D. S.; SPORNS, O. Network neuroscience. Nature Neuroscience, v. 20, n. 3, p. 353-364, 23 mar. 2017.

CASTELLS, M. A sociedade em rede. 6th. ed. Sao Paulo: Paz e Terra, 2002.

CONTI, P. et al. How to reduce the likelihood of coronavirus-19 (CoV-19 or SARS-CoV-2) infection and lung inflammation mediated by IL-1. Journal of biological regulators and homeostatic agents, v. 34, n. 2, 2020.

CONTROL, C. FOR D. Vaccine Testing and Approval Process. Disponível em: <https://www.cdc.gov/vaccines/basics/test-approve.html>. Acesso em: 16 ago. 2020a.

CONTROL, E. C. FOR D. COVID-19 situation update worldwide, as of 29 July 2020. [s.l: s.n.]. Disponível em: <https://www.ecdc.europa.eu/en/geographical-distribution-2019-ncov-cases>.

CUCINOTTA, D.; VANELLI, M. WHO Declares COVID-19 a Pandemic. Acta bio-medica : Atenei Parmensis, v. 91, n. 1, p. 157-160, 2020.

CUNNINGHAM, A. L. et al. Vaccine development: From concept to early clinical testing. Vaccine, $v$. 34, n. 52, p. 6655-6664, dez. 2016.

FIDLER, D. P. Negotiating Equitable Access to Influenza Vaccines: Global Health Diplomacy and the Controversies Surrounding Avian Influenza H5N1 and Pandemic Influenza H1N1. In: ROSSKAM, E.; KICKBUSCH, I. (Eds.). . Negotiating and Navigating Global Health. [s.I.] World Scientific, 2011. p. $161-172$. 
GIUNTA, E. E. et al. Storiepossibili (possible stories): Measuring social networks and designing scenarios to address new urban questions. Strategic Design Research Journal, v. 10, n. 3, 27 nov. 2017.

HAMED, M. A. An overview on COVID-19: reality and expectation. Bulletin of the National Research Centre, v. 44, n. 1, p. 86, 1 dez. 2020.

HOLSTON, D. The Strategic Designer: Tools \& Techniques for Managing the Design Process. Avon, MA: Simon and Schuster, 2011.

KWOK, K. O. et al. Herd immunity - estimating the level required to halt the COVID-19 epidemics in affected countries. The Journal of infection, v. 80, n. 6, p. e32-e33, 2020.

LERMA, B.; DAL PALÙ, D.; DE GIORGI, C. Questões avançadas de design: uma abordagem estratégica e investigativa para projetos sem mercado. Strategic Design Research Journal, v. 7, n. 3, 8 jul. 2015.

MANZINI, E.; JÉGOU, F. Design degli scenari. In: Design Multiverso | Notas de fenomenologia do design. POLI ed. [s.l.] Milano, 2006. p. 189-207.

MERONI, A. Strategic design: where are we now? Reflection around the foundations of a recent discipline. Strategic Design Research Journal, v. 1, n. 1, p. 31-38, 1 dez. 2008.

MIELE, V. et al. Nine quick tips for analyzing network data. PLOS Computational Biology, v. 15, n. 12, p. e1007434, 19 dez. 2019.

PARTRIDGE, J. Government to develop $£ 100 \mathrm{~m}$ Covid-19 vaccine manufacturing centre. The Guardian, 2020.

PRONKER, E. S. et al. Risk in Vaccine Research and Development Quantified. PLoS ONE, v. 8, n. 3, p. e57755, 20 mar. 2013.

SAÚDE, S. DE V. EM. Painel Coronavírus. Disponível em: <https://covid.saude.gov.br/>. Acesso em: 16 ago. 2020.

SCHAFFER DEROO, S.; PUDALOV, N. J.; FU, L. Y. Planning for a COVID-19 Vaccination Program. JAMA, v. 323, n. 24, p. 2458, 23 jun. 2020.

SCHÖN, D. A. Educando o Profissional Reflexivo. Porto Alegre: Artmed, 2000.

SHARPE, H. R. et al. The early landscape of coronavirus disease 2019 vaccine development in the UK and rest of the world. Immunology, v. 160, n. 3, p. 223-232, 2020.

THANH LE, T. et al. The COVID-19 vaccine development landscape. Nature Reviews Drug Discovery, v. 19, n. 5, p. 305-306, 9 maio 2020.

YAMEY, G. et al. Ensuring global access to COVID-19 vaccines. The Lancet, v. 395, n. 10234, p. 1405-1406, maio 2020.

ZURLO, F. Design strategico. Gli spazi ed. Roma: XXI Secolo, 2010. 